Research Article

\title{
Estimation of Generalized Gompertz Distribution Parameters under Ranked-Set Sampling
}

\author{
Mohammed Obeidat $\mathbb{I D}^{1,2}$ Amjad Al-Nasser, ${ }^{3}$ and Amer I. Al-Omari ${ }^{4}$ \\ ${ }^{1}$ Department of Quantitative Methods, King Faisal University, Hofuf, Saudi Arabia \\ ${ }^{2}$ Department of Statistics, Yarmouk University, Irbid, Jordan \\ ${ }^{3}$ College of Business Administration, Al Falah University, Dubai, UAE \\ ${ }^{4}$ Department of Mathematics, Faculty of Science, Al al-Bayt University, Jordan
}

Correspondence should be addressed to Mohammed Obeidat; mobeidat@kfu.edu.sa

Received 2 April 2020; Revised 12 July 2020; Accepted 29 July 2020; Published 7 September 2020

Academic Editor: Ramón M. Rodríguez-Dagnino

Copyright $\odot 2020$ Mohammed Obeidat et al. This is an open access article distributed under the Creative Commons Attribution License, which permits unrestricted use, distribution, and reproduction in any medium, provided the original work is properly cited.

\begin{abstract}
This paper studies estimation of the parameters of the generalized Gompertz distribution based on ranked-set sample (RSS). Maximum likelihood (ML) and Bayesian approaches are considered. Approximate confidence intervals for the unknown parameters are constructed using both the normal approximation to the asymptotic distribution of the ML estimators and bootstrapping methods. Bayes estimates and credible intervals of the unknown parameters are obtained using differential evolution Markov chain Monte Carlo and Lindley's methods. The proposed methods are compared via Monte Carlo simulations studies and an example employing real data. The performance of both ML and Bayes estimates is improved under RSS compared with simple random sample (SRS) regardless of the sample size. Bayes estimates outperform the ML estimates for small samples, while it is the other way around for moderate and large samples.
\end{abstract}

\section{Introduction}

Gompertz distribution was introduced by Gompertz [1] to describe human mortality and to establish actuarial tables. It was also found to be useful in medical sciences because it gives a good fit to data coming from clinical trials on ordered subjects [2]. Gompertz distribution has been extensively studied in the literature (see, for example, El-Din et al. [3] and the reference therein). This paper focuses on the threeparameter generalized Gompertz distribution, which was proposed by El-Gohary et al. [4].

A random variable $X$ is said to have a generalized Gompertz (GG) distribution with parameter vector $\theta=(\lambda, c, \theta)$, denoted as $X: \mathrm{GG}(\lambda, c, \theta)$, if its probability density function and its distribution function are given by

$$
\begin{aligned}
& f(x ; \lambda, c, \theta)=\theta \lambda e^{c x} e^{-\lambda / c\left(e^{c x}-1\right)}\left[1-e^{-\lambda / c\left(e^{c x}-1\right)}\right]^{\theta-1}, \quad x>0, \lambda, \theta>0, c \geq 0, \\
& F(x ; \lambda, c, \theta)=\left[1-e^{-\lambda / c\left(e^{c x}-1\right)}\right]^{\theta}, \quad x>0, \lambda, \theta>0, c \geq 0 .
\end{aligned}
$$

The GG distribution covers the generalized exponential distribution and the one-parameter exponential distribution as special cases when $c$ goes to zero and $\theta=1$. It also covers the Gompertz distribution when $\theta=1$. The GG distribution takes different shapes of the failure rate curve, namely, increasing, constant, decreasing, or bathtub depending on the value of $\theta$ (the shape parameter). The GG distribution is considered as a strong candidate 
distribution for the analysis of reliability data [4] and survival data [5].

Demir and Saracoglu [6] studied maximum likelihood estimation of the GG distribution parameters under progressively type II censored data. Ahmed [7] studied maximum likelihood and Bayesian estimation of the lifetime parameters of the GG distribution under progressively type II censored data. Based on the GG distribution, Borges [5] developed a regression model for survival data. The author proposed an Expectation-Maximization algorithm to estimate the regression parameters. Abu-Zinadah and Al-Oufi [8] studied the estimation of the GG parameters under complete sample using ML, least-squares, weighted leastsquares, and percentiles estimation methods. Estimation of the GG parameters under type II censored sample was studied by Abu-Zinadah and Al-Oufi [9]. The use of GG distribution in lifetime data in the presence of cure fraction, censored data, and covariates was studied by Martinez [10].

Different from the above, this article discusses ML and Bayesian parameter estimation of the GG distribution under ranked-set sampling (RSS) scheme. RSS is a sampling scheme proposed by McIntyre [11] in the hope of improving the estimation of the population mean. RSS is very useful in situations where precise measurements of sample units are difficult, due to high cost or time consumption, but a set of sample units can be accurately ranked at negligible cost or time. For situations where RSS techniques have been found applicable, see Barnett and Moore [12], Wolfe [13], and Frey and Zhang [14].

RSS sampling scheme aims to collect observations from a population that are more representative of it than other probability sampling techniques such as the simple random sampling (SRS) based on the same number of the collected observations. To implement RSS of $n$ observations from a population, follow the following steps:

(i) Select $m$ SRSs of size $m$ each, where $m$ is chosen to be small.

(ii) Rank the units in each sample from smallest to largest. Ranking is done without actually measuring the units with respect to the variable of interest.

(iii) Actual measurements are taken only on the $i^{\text {th }}$ largest unit in the $i^{\text {th }}$ sample, $i=1, \ldots, m$.

(iv) Repeat the previous steps $r$ times (cycles) such that $n=r \times m$.
The resulted sample is denoted as $X_{(i: m) j}$, the $i^{\text {th }}$ largest unit in a set of size $m$ in the $j^{\text {th }}$ cycle, where $i=1, \ldots, m$ and $j=1, \ldots, r$. Based on the above steps, the joint pdf of a RSS is given by the following equation (see Arnold et al. [15]):

$$
\begin{aligned}
f_{X_{(i: m) j}}\left(x_{(i: m) j}\right)= & \prod_{j=1}^{r} \prod_{i=1}^{m} \frac{m !}{(i-1) !(m-i) !} F^{i-1}\left(x_{(i: m) j}\right) \\
& \cdot\left[1-F\left(x_{(i: m) j}\right)\right]^{(m-i)} f\left(x_{(i: m) j}\right),
\end{aligned}
$$

where $\mathbf{x}_{(i: m) j}=\left\{x_{(i: m) j}, i=1, \ldots, m, j=1, \ldots, r\right\}$ and $f$ and $F$ are the pdf and cdf of a random variable $X$.

The remainder of this paper is organized as follows. Section 2 discusses ML estimation and confidence intervals of the model parameters under both RSS and SRS. Bayesian estimators along with credible intervals of the parameters are discussed in Section 3. Section 4 compares between ML and Bayesian method through a Monte Carlo simulation study. Real data example is considered in Section 5 to implement the proposed methods. Section 6 concludes the paper.

\section{Maximum Likelihood Estimation}

This section discusses the parameter estimation of the GG distribution using the ML estimation method under RSS. Moreover, interval estimation of the parameters is discussed based on the observed Fisher information matrix and the normal approximation to the asymptotic distribution of MLEs. Bootstrapping confidence intervals are also considered as an alternative to the normal approximation approach. For comparative purposes, the ML and interval estimation under SRS are investigated.

2.1. MLE under RSS. Let $\left(X_{(1: m) 1}, X_{(2: m) 1}, \ldots, X_{(m: m) 1}\right.$, $\left.\ldots, X_{(1: m) r}, X_{(2: m) r}, \ldots, X_{(m: m) r}\right)$ be a RSS from $\mathrm{GG}(\lambda$, $c, \theta)$, with pdf given in 1 . Let the vector

$$
\mathbf{x}=\left(x_{(1: m) 1}, x_{(2: m) 1}, \ldots, x_{(m: m) 1}, \ldots, x_{(1: m) r}, x_{(2: m) r}, \ldots, x_{(m: m) r}\right)
$$

be the vector of realizations. Substituting (1) and (2) in (3), the likelihood and the log-likelihood functions of a RSS from GG distribution will be as follows:

$$
\begin{aligned}
L_{\mathrm{RSS}}(\lambda, c, \theta ; \mathbf{x})= & \theta^{n} \lambda^{n} \exp \left\{c \sum_{j=1}^{r} \sum_{i=1}^{m} x_{(i: m) j}\right\} \times \prod_{j=1}^{r} \prod_{i=1}^{m} \psi_{i j}\left\{\frac{m !}{(i-1) !(m-i) !}\left[1-\psi_{i j}\right]^{\theta i-1}\left[1-\left(1-\psi_{i j}\right)^{\theta}\right]^{m-i}\right\}, \\
\ell_{\mathrm{RSS}}(\lambda, c, \theta ; \mathbf{x})= & n \log \theta+n \log \lambda+c \sum_{j=1}^{r} \sum_{i=1}^{m} x_{(i: m) j}-\frac{\lambda}{c} \sum_{j=1}^{r} \sum_{i=1}^{m}\left(e^{c x_{(i: m) j}}-1\right)+\log \prod_{j=1}^{r} \prod_{i=1}^{m}\left(\begin{array}{c}
m \\
i
\end{array}\right) \\
& +\sum_{j=1}^{r} \sum_{i=1}^{m}(\theta i-1) \log \left[1-\psi_{i j}\right]+\sum_{j=1}^{r} \sum_{i=1}^{m}(m-i) \log \left[1-\left(1-\psi_{i j}\right)^{\theta}\right],
\end{aligned}
$$


respectively, where $\psi_{\mathrm{ij}}=\exp \left\{-(\lambda / c)\left(e^{c x_{(i: m) j}}-1\right)\right\}$. The first partial derivatives of $\ell_{\mathrm{RSS}}(\lambda, c, \theta, \mathbf{x})$ are as follows:

$$
\begin{aligned}
\frac{\partial \ell_{R S S}}{\partial \theta}= & \frac{n}{\theta}+\sum_{j=1}^{r} \sum_{i=1}^{m} i \log \left[1-\psi_{i j}\right]-\sum_{j=1}^{r} \sum_{i=1}^{m}(m-i) \frac{\left(1-\psi_{i j}\right)^{\theta} \log \left(1-\psi_{i j}\right)}{1-\left(1-\psi_{i j}\right)^{\theta}}, \\
\frac{\partial \ell_{\mathrm{RSS}}}{\partial \lambda}= & \frac{n}{\lambda}-\frac{1}{c} \sum_{j=1}^{r} \sum_{i=1}^{m}\left(e^{c x_{(i: m) j}}-1\right)+\sum_{j=1}^{r} \sum_{i=1}^{m}(\theta i-1) \frac{1 / c\left(e^{c x_{(i: m) j}}-1\right) \psi_{i j}}{1-\psi_{i j}} \\
& -\sum_{j=1}^{r} \sum_{i=1}^{m}(m-i) \frac{\theta\left(1-\psi_{i j}\right)^{\theta-1} 1 / c\left(e^{c x_{(i: m) j}}-1\right) \psi_{i j}}{1-\left(1-\psi_{i j}\right)^{\theta}} \\
\frac{\partial \ell_{\mathrm{RSS}}=}{\partial c} & \sum_{j=1}^{r} \sum_{i=1}^{m} x_{(i: m) j}-\left(\frac{\lambda}{c} \sum_{j=1}^{r} \sum_{i=1}^{m} x_{(i: m) j} e^{c x_{(i: m) j}}-\frac{\lambda}{c^{2}} \sum_{j=1}^{r} \sum_{i=1}^{m}\left(e^{c x_{(i: m) j}}-1\right)\right) \\
& +\sum_{j=1}^{r} \sum_{i=1}^{m}(\theta i-1) \frac{\left(\lambda / c x_{(i: m) j} e^{c x_{(i: m) j}}-\left(\lambda / c^{2}\right)\left(e^{c x_{(i: m) j}}-1\right)\right) \psi_{i j}}{1-\psi_{i j}} \\
& -\sum_{j=1}^{r} \sum_{i=1}^{m}(m-i) \frac{\theta\left(1-\psi_{i j}\right)^{\theta-1}\left(\lambda / c x_{(i: m) j} e^{c x_{(i: m) j}}-\left(\lambda / c^{2}\right)\left(e^{c x_{(i: m) j}}-1\right)\right) \psi_{i j}}{1-\left(1-\psi_{i j}\right)^{\theta}}
\end{aligned}
$$

The MLEs for the parameters $\lambda, c$, and $\theta$ are obtained by maximizing the likelihood in (4) or equivalently by maximizing the log-likelihood in (5). This can be accomplished by setting the partial derivatives in (6)-(8) equal to zero and solving these equations simultaneously. It can be seen that these equations do not have closed-form solutions; therefore, the Newton-Raphson method is used to obtain the estimates. The algorithm is comprised of the following steps:

Step 1: start with an initial guess $\left(\lambda^{(0)}, c^{(0)}, \theta^{(0)}\right)$ as a starting point of the iterations.

Step 2: at iteration $k \geq 1$, do the following:

Evaluate the gradients $S(\lambda, c, \theta)=\left(\partial \ell_{\mathrm{RSS}} / \partial \lambda\right.$, $\left.\partial \ell_{\mathrm{RSS}} / \partial c, \partial \ell_{\mathrm{RSS}} / \partial \theta\right)$ at $\left(\lambda^{(k-1)}, c^{(k-1)}, \theta^{(k-1)}\right)$.

Evaluate the observed Fisher information matrix, denoted by $I(\lambda, c, \theta)$, at $\left(\lambda^{(k-1)}, c^{(k-1)}, \theta^{(k-1)}\right)$. The Fisher information matrix will be defined later in this section.

Update the parameter vector by

$$
\begin{aligned}
& \left(\lambda^{(k)}, c^{(k)}, \theta^{(k)}\right) \\
& =\left(\lambda^{(k-1)}, c^{(k-1)}, \theta^{(k-1)}\right)+S\left(\lambda^{(k-1)}, c^{(k-1)}, \theta^{(k-1)}\right) \\
& \quad \times I^{-1}\left(\lambda^{(k-1)}, c^{(k-1)}, \theta^{(k-1)}\right),
\end{aligned}
$$

where $I^{-1}$ stands for the inverse of the Fisher information.

Step 3: repeat Step 2 until the absolute difference between $\left(\lambda^{(k)}, c^{(k)}, \theta^{(k)}\right)$ and $\left(\lambda^{(k-1)}, c^{(k-1)}, \theta^{(k-1)}\right)$ is less than a threshold value, usually taken to be $10^{-6}$.
Step 4: the MLEs of $(\lambda, c, \theta)$ are the parameter values at the last iteration, denote them by $\widehat{\theta}_{\mathrm{ML}}=\left(\widehat{\lambda}_{\mathrm{ML}}, \widehat{c}_{\mathrm{ML}}\right.$, $\left.\widehat{\theta}_{\mathrm{ML}}\right)$.

To obtain confidence intervals of the parameters, the asymptotic properties of the MLE were used. The MLE is asymptotically normal with mean equal to the true parameter values and variance-covariance matrix equal to the inverse of the observed Fisher information matrix (see Lawless [16]). Observed Fisher information matrix is defined to be the matrix of the second partial derivatives of the negative log-likelihood with respect to the model parameters, that is

$$
\widehat{\boldsymbol{\theta}}_{\mathrm{ML}} \sim \operatorname{MVN}\left(\boldsymbol{\theta}, I^{-1}(\lambda, c, \theta)\right)
$$

where

$$
I(\lambda, c, \theta)=-\left(\begin{array}{ccc}
\frac{\partial^{2} \ell_{\mathrm{RSS}}}{\partial \lambda^{2}} & \frac{\partial^{2} \ell_{\mathrm{RSS}}}{\partial \lambda \partial c} & \frac{\partial^{2} \ell_{\mathrm{RSS}}}{\partial \lambda \partial \theta} \\
\frac{\partial^{2} \ell_{\mathrm{RSS}}}{\partial \lambda \partial c} & \frac{\partial^{2} \ell_{\mathrm{RSS}}}{\partial c^{2}} \frac{\partial^{2} \ell_{\mathrm{RSS}}}{\partial c \partial \theta} \\
\frac{\partial^{2} \ell_{\mathrm{RSS}}}{\partial \lambda \partial \theta} & \frac{\partial^{2} \ell_{\mathrm{RSS}}}{\partial c \partial \theta} & \frac{\partial^{2} \ell_{\mathrm{RSS}}}{\partial \theta^{2}}
\end{array}\right),
$$

therefore, a $(1-\alpha) 100 \%$ confidence intervals of the model parameters are $\left(\widehat{\lambda}_{\mathrm{ML}} \pm Z_{\alpha / 2} \sqrt{\left.\widehat{\operatorname{var}}\left(\hat{\lambda}_{\mathrm{ML}}\right)\right)}, \quad\left(\widehat{c}_{\mathrm{ML}} \pm Z_{\alpha / 2}\right.\right.$ $\left.\sqrt{\widehat{\operatorname{var}}\left(\widehat{c}_{\mathrm{ML}}\right)}\right)$, and $\left(\widehat{\theta}_{\mathrm{ML}} \pm Z_{\alpha / 2} \sqrt{\left.\widehat{\operatorname{var}}\left(\widehat{\theta}_{\mathrm{ML}}\right)\right)}\right.$, where $\widehat{\operatorname{var}}\left(\widehat{\lambda}_{\mathrm{ML}}\right), \widehat{\operatorname{var}}\left(\widehat{c}_{\mathrm{ML}}\right)$, and $\widehat{\operatorname{var}}\left(\widehat{\theta}_{\mathrm{ML}}\right)$ are the diagonal elements 
4

Journal of Probability and Statistics

of $I^{-1}(\lambda, c, \theta)$, and $Z_{\alpha / 2}$ is the $\alpha / 2$ upper quartile of the standard normal distribution.
The second partial derivatives of $\ell_{\mathrm{RSS}}(\lambda, c, \theta, \mathbf{x})$ that are the elements of $I(\lambda, c, \theta)$ are as follows:

$$
\begin{aligned}
& \frac{\partial^{2} \ell_{\mathrm{RSS}}}{\partial \theta^{2}}=-\frac{n}{\theta^{2}}-\sum_{j=1}^{r} \sum_{i=1}^{m} \frac{(m-i)\left[\log \left(1-\psi_{i j}\right)\right]^{2}}{\left[1-\left(1-\psi_{i j}\right)^{\theta}\right]^{2}}\left\{\left[1-\left(1-\psi_{i j}\right)^{\theta}\right]\left(1-\psi_{i j}\right)^{\theta} \times+\left(1-\psi_{i j}\right)^{2 \theta}\right\}, \\
& \frac{\partial^{2} \ell_{\mathrm{RSS}}}{\partial \theta \partial \lambda}=\sum_{j=1}^{r} \sum_{i=1}^{m} i \frac{1 / c\left(e^{c x_{(i: m) j}}-1\right) \psi_{i j}}{1-\psi_{i j}}-\sum_{j=1}^{r} \sum_{i=1}^{m} \frac{(m-i)}{\left[1-\left(1-\psi_{i j}\right)^{\theta}\right]^{2}} \\
& \times\left\{\left[1-\left(1-\psi_{i j}\right)^{\theta}\right]\left[\frac{1}{c}\left(e^{c x_{(i: m) j}}-1\right) \psi_{i j}\left(1-\psi_{i j}\right)^{\theta-1}\left(\theta \log \left(1-\psi_{i j}\right)+1\right)\right]+\left(1-\psi_{i j}\right)^{\theta} \log \left(1-\psi_{i j}\right)\left[\theta\left(1-\psi_{i j}\right)^{\theta-1} \frac{1}{c}\left(e^{c x_{(i: m) j}}-1\right) \psi_{i j}\right]\right\}, \\
& \frac{\partial^{2} \ell_{\mathrm{RSS}}}{\partial \lambda^{2}}=-\frac{n}{\lambda}+\sum_{j=1}^{r} \sum_{i=1}^{m}(\theta i-1) \frac{1 / c \psi_{i j}\left(e^{c x_{(i: m) j}}-1\right)^{2}}{\left(1-\psi_{i j}\right)^{2}}\left\{\left(1-\psi_{i j}\right) \frac{-1}{c}-\psi_{i j} \frac{1}{c}\right\} \\
& -\sum_{j=1}^{r} \sum_{i=1}^{m} \frac{(m-i) \theta 1 / c\left(e^{c x_{(i: m) j}}-1\right)}{\left(1-\left(1-\psi_{i j}\right)^{\theta}\right)^{2}}\left\{( 1 - ( 1 - \psi _ { i j } ) ^ { \theta } ) \left(\left(\left(1-\psi_{i j}\right)^{\theta-1-1} \frac{1}{c}\left(e^{c x_{(i: m) j}}-1\right) \psi_{i j}+\psi_{i j}(\theta-1)\left(1-\psi_{i j}\right)^{\theta-2} \frac{1}{c}\left(e^{c x_{(i: m) j}}-1\right) \psi_{i j}\right)\right.\right. \\
& \left.+\left(1-\psi_{i j}\right)^{2(\theta-1)} e^{-2 \lambda / c\left(e^{c x(i: m) j}-1\right)} \theta \frac{1}{c}\left(e^{c x_{c x}(i: m) j}-1\right)\right\}, \\
& \frac{\partial^{2} \ell_{\mathrm{RSS}}}{\partial c \partial \lambda}=-\left(\frac{1}{c} \sum_{j=1}^{r} \sum_{i=1}^{m} x_{(i: m) j} e^{c x_{(i: m) j}}-\frac{1}{c^{2}} \sum_{j=1}^{r} \sum_{i=1}^{m}\left(e^{c x_{(i: m) j}}-1\right)\right)+\sum_{j=1}^{r} \sum_{i=1}^{m} \frac{(\theta i-1)}{\left(1-\psi_{i j}\right)^{2}} \\
& \times\left\{\left(1-\psi_{i j}\right)\left[\left(\frac{\lambda}{c} x_{(i: m) j} e^{c x_{(i: m) j}}-\frac{\lambda}{c^{2}}\left(e^{c x_{(i: m) j}}-1\right)\right) \frac{-1}{c}\left(e^{c x_{(i: m) j}}-1\right) \psi_{i j}+\left(\frac{1}{c} x_{(i: m) j} e^{c x_{(i: m) j}}-\frac{1}{c^{2}}\left(e^{c x_{(i: m) j}}-1\right)\right) \psi_{i j}\right]\right. \\
& \left.-\left(\frac{\lambda}{c} x_{(i: m) j} e^{c x_{(i: m) j}}-\frac{\lambda}{c^{2}}\left(e^{c x_{(i: m) j}}-1\right)\right) \frac{1}{c}\left(e^{c x_{(i: m) j}}-1\right) e^{-2 \lambda / c\left(e^{c x(i: m) j}-1\right)}\right\} \\
& -\sum_{j=1}^{r} \sum_{i=1}^{m} \frac{(m-i) \theta}{\left(1-\left(1-\psi_{i j}\right)^{\theta}\right)^{2}}\left\{\left(1-\left(1-\psi_{i j}\right)^{\theta}\right)\right. \\
& \times\left[\left(1-\psi_{i j}\right)^{\theta-1}\left(\left(\frac{\lambda}{c} x_{(i: m) j} e^{c x_{(i: m) j}}-\frac{\lambda}{c^{2}}\left(e^{c x_{(i: m) j}}-1\right)\right) \frac{-1}{c}\left(e^{c x_{(i: m) j}}-1\right) \psi_{i j}+\psi_{i j}\left(\frac{1}{c} x_{(i: m) j} e^{c x_{(i: m) j}}-\frac{1}{c^{2}}\left(e^{c x_{(i: m) j}}-1\right)\right)\right)\right. \\
& \left.+(\theta-1) \frac{1}{c}\left(e^{c x_{(i: m) j}}-1\right) e^{-(2 \lambda / c)\left(e^{c x(i: m) j}-1\right)}\left(1-\psi_{i j}\right)^{\theta-2}\left(\frac{\lambda}{c} x_{(i: m) j} e^{c x_{(i: m) j}}-\frac{\lambda}{c^{2}}\left(e^{c x_{(i: m) j}}-1\right)\right)\right] \\
& \left.+\theta \frac{1}{c}\left(e^{c x_{(i: m) j}}-1\right) e^{-2 \lambda / c\left(e^{c x_{(i: m) j}}-1\right)}\left(1-\psi_{i j}\right)^{2(\theta-1)}\left(\frac{\lambda}{c} x_{(i: m) j} e^{c x_{(i: m) j}}-\frac{\lambda}{c^{2}}\left(e^{c x_{(i: m) j}}-1\right)\right)\right\}, \\
& \frac{\partial^{2} \ell_{R S S}}{\partial c^{2}}=-\left(\frac{\lambda}{c} \sum_{j=1}^{r} \sum_{i=1}^{m} x_{(i: m) j}^{2} e^{c x_{(i: m) j}}-2 \frac{\lambda}{c^{2}} \sum_{j=1}^{r} \sum_{i=1}^{m} x_{(i: m) j} e^{c x_{(i: m) j}}+2 \frac{\lambda}{c^{3}} \sum_{j=1}^{r} \sum_{i=1}^{m}\left(e^{c x_{(i: m) j}}-1\right)\right) \\
& +\sum_{j=1}^{r} \sum_{i=1}^{m} \frac{(\theta i-1)}{\left(1-\psi_{i j}\right)^{2}}\left\{\left(1-\psi_{i j}\right)\left[(-1)\left(\frac{\lambda}{c} x_{(i: m) j} e^{c x_{(i: m) j}}-\frac{\lambda}{c^{2}}\left(e^{c x_{(i: m) j}}-1\right)\right)^{2}+\left(\frac{\lambda}{c} x_{(i: m) j}^{2} e^{c x_{(i: m) j}}-2 \frac{\lambda}{c^{2}} x_{(i: m) j} e^{c x_{(i: m) j}}+\frac{2 \lambda}{c^{3}}\left(e^{c x_{(i: m) j}}-1\right)\right)\right] \psi_{i j}\right. \\
& \left.-\left(\frac{\lambda}{c} x_{(i: m) j} e^{c x_{(i: m) j}}-\frac{\lambda}{c^{2}}\left(e^{c x_{(i: m) j}}-1\right)\right)^{2} e^{-2 \lambda / c\left(e^{c x(i: m) j}-1\right)}\right\} \\
& -\sum_{j=1}^{r} \sum_{i=1}^{m} \frac{(m-i) \theta}{\left(1-\left(1-\psi_{i j}\right)^{\theta}\right)^{2}}\left\{( 1 - ( 1 - \psi _ { i j } ) ^ { \theta } ) \left\{\left(1-\psi_{i j}\right)^{\theta-1} \times\right.\right.
\end{aligned}
$$




$$
\begin{aligned}
& {\left[(-1)\left(\frac{\lambda}{c} x_{(i: m) j} e^{c x_{(i: m) j}}-\frac{\lambda}{c^{2}}\left(e^{c x_{(i: m) j}}-1\right)\right)^{2}+\left(\frac{\lambda}{c} x_{(i: m) j}^{2} e^{c x_{(i: m) j}}-2 \frac{\lambda}{c^{2}} x_{(i: m) j} e^{c x_{(i: m) j}}+2 \frac{\lambda}{c^{3}}\left(e^{c x_{(i: m) j}}-1\right)\right) \psi_{i j}\right]} \\
& \left.+(\theta-1)\left(1-\psi_{i j}\right)^{\theta-2}\left(\frac{\lambda}{c} x_{(i: m) j} e^{c x_{(i: m) j}}-\frac{\lambda}{c^{2}}\left(e^{c x_{(i: m) j}}-1\right)\right)^{2} e^{-2 \lambda / c\left(e^{c x_{(i: m) j}}-1\right)}\right\} \\
& \left.+\theta\left(1-\psi_{i j}\right)^{2(\theta-1)}\left(\frac{\lambda}{c} x_{(i: m) j} e^{c x_{(i: m) j}}-\frac{\lambda}{c^{2}}\left(e^{c x_{(i: m) j}}-1\right)\right) e^{-2 \lambda / c\left(e^{c x_{(i: m) j}}-1\right)}\right\} .
\end{aligned}
$$

2.2. MLE under SRS. Let $X_{1}, X_{2}, \ldots, X_{n}$ be a SRS from $\mathrm{GG}(\lambda, c, \theta)$, with pdf given in 1 , where $\mathbf{x}=\left(x_{1}, x_{2}, \ldots, x_{n}\right)$ being the vector of realizations. The likelihood and the log-likelihood functions are given by

$$
\begin{aligned}
L_{\mathrm{SRS}}(\lambda, c, \theta ; \mathbf{x}) & =\theta^{n} \lambda^{n} \exp \left\{c \sum_{i=1}^{n} x_{i}\right\} \exp \left\{-\frac{\lambda}{c} \sum_{i=1}^{n}\left(e^{c x_{i}}-1\right)\right\} \prod_{i=1}^{n}\left[1-\psi_{i}\right]^{\theta-1}, \\
\ell_{\mathrm{SRS}}(\lambda, c, \theta ; \mathbf{x}) & =\log L(\lambda, c, \theta, \mathbf{x}) \\
& =n \log \theta+n \log \lambda+c \sum_{i=1}^{n} x_{i}-\frac{\lambda}{c} \sum_{i=1}^{n}\left(e^{c x_{i}}-1\right)+(\theta-1) \sum_{i=1}^{n} \log \left[1-\psi_{i}\right],
\end{aligned}
$$

respectively, where $\psi_{i}=e^{-(\lambda / c)\left(e^{c x_{i}}-1\right)}$. The first partial derivatives of $\ell_{\mathrm{SRS}}(\lambda, c, \theta, \mathbf{x})$ are as follows:

$$
\begin{aligned}
\frac{\partial \ell_{\mathrm{SRS}}}{\partial \theta}= & \frac{n}{\theta}+\sum \log \left(1-\psi_{i}\right) \\
\frac{\partial \ell_{\mathrm{SRS}}}{\partial \lambda}= & \frac{n}{\lambda}-\frac{1}{c} \sum\left(e^{c x_{i}}-1\right)+(\theta-1) \sum \frac{1 / c\left(e^{c x_{i}}-1\right) \psi_{i}}{\left[1-\psi_{i}\right]} \\
\frac{\partial \ell_{\mathrm{SRS}}}{\partial c}= & \sum x_{i}-\frac{\lambda}{c^{2}}\left(c \sum x_{i} e^{c x_{i}}-\sum\left(e^{c x_{i}}-1\right)\right) \\
& +(\theta-1) \sum \frac{\left(\lambda / c x_{i} e^{c x_{i}}-\left(\lambda / c^{2}\right)\left(e^{c x_{i}}-1\right)\right) \psi_{i}}{\left[1-\psi_{i}\right]}
\end{aligned}
$$

$$
\begin{aligned}
\frac{\partial^{2} \ell_{\mathrm{SRS}}}{\partial \theta^{2}}= & -\frac{n}{\theta^{2}}, \\
\frac{\partial^{2} \ell_{\mathrm{SRS}}}{\partial \theta \partial \lambda}= & \sum \frac{1 / c\left(e^{c x_{i}}-1\right) \psi_{i}}{\left[1-\psi_{i}\right]}, \\
\frac{\partial^{2} \ell_{\mathrm{SRS}}}{\partial \theta \partial c}= & \sum \frac{\left(\lambda / c x_{i} e^{c x_{i}}-\lambda / c^{2}\left(e^{c x_{i}}-1\right)\right) \psi_{i}}{\left[1-\psi_{i}\right]} \\
\frac{\partial^{2} \ell_{\mathrm{SRS}}}{\partial \lambda \partial c}= & -\frac{1}{c^{2}}\left(c \sum x_{i} e^{c x_{i}}-\sum\left(e^{c x_{i}}-1\right)\right)+(\theta-1) \sum\left\{\left(\frac{1}{c} x_{i} e^{c x_{i}}-\frac{1}{c^{2}}\left(e^{c x_{i}}-1\right)\right)\right. \\
& \left.\times\left[\left[1-\psi_{i}\right]\left(\lambda\left(\frac{-1}{c}\right)\left(e^{c x_{i}}-1\right) \psi_{i}+\psi_{i}\right) \lambda\left(\psi_{i}\right)\left(\frac{1}{c}\left(e^{c x_{i}}-1\right) \psi_{i}\right)\right] \frac{1}{\left[1-\psi_{i}\right]^{2}}\right\},
\end{aligned}
$$

The MLEs of the model parameters based on SRS are obtained by setting the partial derivatives in (16)-(18) equal to zero. Clearly, this system of equations does not have closed-form solution; therefore, Newton-Raphson method will be used.

The second partial derivatives of $\ell_{\mathrm{SRS}}(\lambda, c, \theta, \mathbf{x})$ that are the elements of $I(\lambda, c, \theta)$ are as follows: 


$$
\begin{aligned}
\frac{\partial^{2} \ell_{\mathrm{SRS}}}{\partial \lambda^{2}}= & -\frac{n}{\lambda^{2}}+(\theta-1) \sum\left\{\frac{1 / c\left(e^{c x_{i}}-1\right)}{\left[1-\psi_{i}\right]^{2}}\left\{\left[1-\psi_{i}\right]\left(\left(\frac{-1}{c}\right)\left(e^{c x_{i}}-1\right) \psi_{i}\right)-\left(\psi_{i}\right)\left(\frac{1}{c}\left(e^{c x_{i}}-1\right) \psi_{i}\right)\right\}\right\}, \\
\frac{\partial^{2} \ell_{\mathrm{SRS}}}{\partial c^{2}}= & \frac{2 \lambda}{c^{3}}\left(c \sum x_{i} e^{c x_{i}}-\sum\left(e^{c x_{i}}-1\right)\right)-\frac{\lambda}{c^{2}} c \sum x_{i}^{2} e^{c x_{i}}+(\theta-1) \sum\left\{\frac{1}{\left[1-\psi_{i}\right]^{2}}\right. \\
& \times\left[1-\psi_{i}\right]\left[(-1)\left(\frac{\lambda}{c} x_{i} e^{c x_{i}}-\frac{\lambda}{c^{2}}\left(e^{c x_{i}}-1\right)\right)^{2} \psi_{i}\right. \\
& \left.\left.+\psi_{i}\left(\frac{\lambda}{c} x_{i}^{2} e^{c x_{i}}-\frac{2 \lambda}{c^{2}} x_{i} e^{c x_{i}}+\frac{2 \lambda}{c^{3}}\left(e^{c x_{i}}-1\right)\right)\right]-\left[\left(\frac{\lambda}{c} x_{i} e^{c x_{i}}-\frac{\lambda}{c^{2}}\left(e^{c x_{i}}-1\right)\right) \psi_{i}\right]^{2}\right\} .
\end{aligned}
$$

2.3. Bootstrap Confidence Interval. Constructing confidence intervals for the model parameters using the normal approximations may not work well when the sample size $n$ is small. Resampling methods are alternative ways that may provide more accurate approximate confidence intervals. One popular resampling method is the bootstrapping method. This section aims to discuss the percentile bootstrap (Boot-p) confidence interval proposed by Efron [17]. The Boot-p confidence interval can be described as follows:

(i) Select a random sample (whether RSS or SRS) from the population and obtain the MLE $\widehat{\theta}$ of the model parameter $\theta$ as discussed in Section 2

(ii) Based on the specified sampling scheme (RSS or SRS), generate a bootstrap random sample from the GG distribution with parameters $\widehat{\theta}$

(iii) Obtain the MLE of the model parameters based on the bootstrap sample and denote this bootstrap estimate by $\hat{\theta}^{*}$

(iv) Repeat the second and third steps above $N$ times to obtain $\widehat{\theta}_{1}^{*}, \ldots, \widehat{\theta}_{N}^{*}$

(v) Arrange the above estimates in ascending order to obtain the ordered estimates $\widehat{\theta}_{(1)}^{*}, \ldots, \widehat{\theta}_{(N)}^{*}$

(vi) A $(1-\alpha) 100 \%$ confidence interval is then obtained from the $100 \alpha / 2$ and $100(1-\alpha / 2)$ empirical percentiles of the bootstrap estimates obtained in the previous step

\section{Bayesian Estimation}

In this section, Bayes' estimates and the Bayesian credible intervals of the parameters $(\lambda, c, \theta)$ are obtained using Markov chain Monte Carlo Methods (MCMCs) under both RSS and SRS.

Two important components of Bayesian analysis are the choice of prior distribution of the parameters and the loss function. The prior distribution reflects the prior knowledge or information about the parameters of interest prior to collecting the data. If there is no such knowledge, then a weakly informative prior could be considered. The loss function measures the loss incurred when estimating a parameter $\theta$ by an estimator $\hat{\theta}$ and is used as a criterion for good estimators.

Independent gamma priors are assumed for the parameters, that is

$$
\pi(\boldsymbol{\theta}) \propto \lambda^{\alpha_{1}-1} e^{-\beta_{1} \lambda} c^{\alpha_{2}-1} e^{-\beta_{2} c} \theta^{\alpha_{3}-1} e^{-\beta_{3} \theta} \lambda, c, \theta>0 .
$$

For the gamma prior to be weakly informative, the hyperparameters $\alpha_{i}$ and $\beta_{i}, i=1,2,3$, are assumed to equal a small value such as 0.001 . Bayesian inference is then obtained based on the posterior distribution, the distribution of the parameters given the data $D$, that is,

$$
\pi(\boldsymbol{\theta} \mid D) \propto L(\boldsymbol{\theta} ; D) \pi(\boldsymbol{\theta})
$$

where $L(\theta ; D)$ is the likelihood function.

In this work, we will use the most widely used loss function in Bayesian inference, that is, the squared error loss (SEL) function, which is given by

$$
L(\boldsymbol{\theta}, \widehat{\boldsymbol{\theta}})=(\boldsymbol{\theta}-\widehat{\boldsymbol{\theta}})^{2} \text {. }
$$

Bayes' estimator of the parameter $\theta$ based on SEL is the posterior mean, that is,

$$
\widehat{\boldsymbol{\theta}}=\frac{\int \boldsymbol{\theta} \pi(\boldsymbol{\theta} \mid D) \mathrm{d} \boldsymbol{\theta}}{\int \pi(\boldsymbol{\theta} \mid D) \mathrm{d} \boldsymbol{\theta}} .
$$

3.1. Bayesian Estimation under RSS. The joint posterior distribution of the parameters $\lambda, c$, and $\theta$ under RSS can be obtained by combining the likelihood in (4) and the prior in (21) via Bayes' theorem. Up to a normalizing constant, it can be written as

$$
\begin{aligned}
\pi(\lambda, c, \theta \mid \mathbf{x})= & \frac{\pi(\lambda, c, \theta) L_{\mathrm{RSS}}(\lambda, c, \theta ; \mathbf{x})}{\iiint \pi(\lambda, c, \theta) L_{\mathrm{RSS}}(\lambda, c, \theta, \mathbf{x}) \mathrm{d} \lambda \mathrm{d} c \mathrm{~d} \theta} \\
& \propto \lambda^{\alpha_{1}-1} e^{-\beta_{1} \lambda} c^{\alpha_{2}-1} e^{-\beta_{2} c} \theta^{\alpha_{3}-1} e^{-\beta_{3} \theta} \theta^{n} \lambda^{n} \\
& \cdot \exp \left\{c \sum_{j=1}^{r} \sum_{i=1}^{m} x_{(i: m) j}\right\} \\
& \times \prod_{j=1}^{r} \prod_{i=1}^{m} \psi_{i j}\left\{\left[1-\psi_{i j}\right]^{\theta i-1}\left[1-\left(1-\psi_{i j}\right)^{\theta}\right]^{m-i}\right\} .
\end{aligned}
$$

Using this posterior, one can obtain Bayes' estimator of any function $g(\lambda, c, \theta)$ of the parameters by finding the posterior mean, that is, 


$$
\begin{aligned}
\widehat{g} & =\iiint g \pi(\lambda, c, \theta \mid \mathbf{x}) \mathrm{d} \lambda \mathrm{d} c \mathrm{~d} \theta \\
& =\frac{\iiint g \pi(\lambda, c, \theta) L_{\mathrm{RSS}}(\lambda, c, \theta ; \mathbf{x}) \mathrm{d} \lambda \mathrm{d} c \mathrm{~d} \theta}{\iiint \pi(\lambda, c, \theta) L_{\mathrm{RSS}}(\lambda, c, \theta, \mathbf{x}) \mathrm{d} \lambda \mathrm{d} c \mathrm{~d} \theta} .
\end{aligned}
$$

Clearly, the posterior distribution involves intractable integrals; this is because the likelihood function based on RSS is complicated. Therefore, a Markov chain Monte Carlo (MCMC) method is proposed to obtain Bayes' estimates of the parameters. MCMC methods aim to generate samples from the joint posterior density function and to use them to compute the Bayes estimate of the parameters of interest. To implement the MCMC methodology, we consider the Metropolis-Hasting (M-H) sampler. The $\mathrm{M}-\mathrm{H}$ sampler is summarized in the following steps:

Step 1: start with initial guess $\left(\lambda^{(0)}, c^{(0)}, \theta^{(0)}\right)$ as a starting point of the $\mathrm{M}-\mathrm{H}$ sampler.

Step 2: choose a proposal kernel from which it is easy to sample from and which have the main characteristics of the posterior. Denote this proposal by $q(\lambda, c, \theta)$.

Step 3: for $k \geq 1$, do the following steps

Sample a proposed realization $\left(\lambda^{(*)}, c^{(*)}, \theta^{(*)}\right)$ from $q(\lambda, c, \theta)$.

Calculate the acceptance ratio

$$
r^{*}=\frac{\pi\left(\lambda^{*}, c^{*}, \theta^{*} \mid \mathbf{x}\right)}{\pi\left(\lambda^{(k-1)}, c^{(k-1)}, \theta^{(k-1)} \mid \mathbf{x}\right)} \frac{q\left(\lambda^{(k-1)}, c^{(k-1)}, \theta^{(k-1)}\right)}{q\left(\lambda^{*}, c^{*}, \theta^{*}\right)}
$$

Set $\left(\lambda^{(k)}, c^{(k)}, \theta^{(k)}\right)=\left(\lambda^{(*)}, c^{(*)}, \theta^{(*)}\right)$ with probability $p=\min \left(1, r^{*}\right)$, and otherwise, set $\left(\lambda^{(k)}, c^{(k)}, \theta^{(k)}\right)=$ $\left(\lambda^{(k-1)}, c^{(k-1)}, \theta^{(k-1)}\right)$.

Step 4: repeat Step 3 for large number of iterations, say $M$, until convergence is assured.

In our simulations, independent normal kernel was used as a proposal distribution. The mean of this proposal is taken to equal to the previously sampled value and standard deviation equal to the square root of the inverse of the observed Fisher information scaled with a factor of $2.38 / \sqrt{d}$, where $d=3$ is the dimension of the parameter space (see Gelman et al. [18]).

The abovementioned $\mathrm{M}-\mathrm{H}$ algorithm is called the Random-Walk M-H (RW-M-H), due to its randomness of proposing a new realization from the posterior. One of the main drawbacks of the RW-M-H algorithm in complex posteriors is its slow convergence due to the dependency between parameters. In our simulations, we noticed that the RW-M-H algorithm did not provide a true nominal coverage of the Bayesian credible interval. Therefore, the differential evolution M-H (DE-M-H) developed by Braak [19] is used to improve the performance of the $\mathrm{M}-\mathrm{H}$ algorithm. Braak [19] stated that the main advantage of the DE-M-H is its ability to handle issues as nonconvergence, colinear parameters, and multimodal densities. The DE-M-H comprises of running multiple chains, say $N$, which are initialized from overdispersed states. The main feature is that the proposed value in each chain uses information from two randomly selected chains from the remaining chains. This allows the chains to learn from each other through the process. The DE-M-H can be implemented as follows. Set $\theta=(\lambda, c, \theta)$.

Step 1: $N$ chains are initialized, $\left(\theta_{1}^{(0)}, \ldots, \theta_{N}^{(0)}\right)$. Braak [19] stated that the algorithm would work well for $N$ between $2 d$ to $3 d$.

Step 2: for $k \geq 1$.

The proposal for the $i^{\text {th }}$ chain, $i=1, \ldots, N$, is $\theta_{i}^{(p)}=\theta_{i}^{(k-1)}+\gamma\left(\theta_{j}^{(k-1)}-\theta_{l}^{(k-1)}\right)+e$.

The proposed value is accepted with probability $\min \left(1, r^{*}\right)$, with

$$
r^{*}=\frac{\pi\left(\boldsymbol{\theta}_{i}^{(p)} \mid \mathbf{x}\right)}{\pi\left(\boldsymbol{\theta}_{i}^{(k-1)} \mid \mathbf{x}\right)}
$$

Step 3: repeat Step 2 for large number of iterations, say M.

For the DE-M-H algorithm, define the following:

$\theta_{i}^{(k-1)}$ is the previous state of the $i^{\text {th }}$ chain.

$\theta_{l}^{(k-1)}$ and $\theta_{j}^{(k-1)}$ are previous states of randomly selected without replacement chains from the remaining chains without the $i^{t h}$ chain. This ensures that the chains are learning from each other.

$e$ is drawn from normal $\left(0, \sigma^{2}\right)$, where $\sigma^{2}$ is chosen to be small. In our simulations, $\sigma$ is taken to equal the standard deviations obtained from the observed Fisher information.

$\gamma$ is a scaling factor used to provide an acceptable acceptance probability. The default choice is $2.38 / \sqrt{d}$ (see Gelman et al. [18]).

The resulted posterior samples can be used to find the posterior estimates and credible intervals as follows. $\widehat{\theta}_{\mathrm{BAY}}=$ $\left(\widehat{\lambda}_{\mathrm{BAY}}, \widehat{c}_{\mathrm{BAY}}, \widehat{\theta}_{\mathrm{BAY}}\right)$, where

$$
\begin{aligned}
\widehat{\lambda}_{\mathrm{BAY}} & =\frac{\sum \sum \lambda_{i}^{(k)}}{M}, \\
\widehat{c}_{\mathrm{BAY}} & =\frac{\sum \sum c_{i}^{(k)}}{M}, \\
\widehat{\theta}_{\mathrm{BAY}} & =\frac{\sum \sum \theta_{i}^{(k)}}{M} .
\end{aligned}
$$

In addition to the point estimator, $\widehat{\theta}_{\mathrm{BAY}}$, of $\theta$, Bayesian credible interval can be obtained from the posterior samples. One popular credible interval is the highest posterior density (HPD) credible interval. The HPD interval can be constructed from the empirical cumulative distribution function (cdf) of the posterior samples as the shortest interval for which the difference in the empirical cdf values of the endpoints is the desired nominal probability. 
3.2. Bayesian Estimation under SRS. The Bayesian approach under SRS is similar to the one under RSS. The only change is that the likelihood $L_{\mathrm{SRS}}$ is used in place of $L_{\mathrm{RSS}}$ in the formula of the posterior distribution in (25). DE-M-H methods were used to obtain posterior samples and making Bayesian inference.

3.3. Lindley's Approximation for Bayesian Estimates. Another way of obtaining Bayesian estimates is by approximating the ratio of integrals in (26). Many attempts have been proposed in the literature to approximate such ratio of integrals. One popular method is proposed by Lindley [20]. Lindley's procedure is outlined as follows.

The ratio of integrals is written in the following form:

$$
\widehat{g}=\frac{\iiint g \exp \{\Lambda(\boldsymbol{\theta})\} \mathrm{d} \lambda \mathrm{d} c \mathrm{~d} \theta}{\iiint \exp \{\Lambda(\boldsymbol{\theta})\} \mathrm{d} \lambda \mathrm{d} c \mathrm{~d} \theta},
$$

where $\Lambda(\theta)=\log (\pi(\theta \mid \mathbf{x}))$ is the $\log$ posterior distribution. By expanding $\Lambda(\theta)$ using Taylor series expansion about the posterior mode $\theta^{*}$, Lindley obtained the Bayes estimator of $g(\theta)$ to be

$$
\widehat{g}=g\left(\boldsymbol{\theta}^{*}\right)+\frac{1}{2} \sum g_{i j}\left(\boldsymbol{\theta}^{*}\right) \tau_{i j}+\frac{1}{2} \sum \Lambda_{i j k} g_{l}\left(\boldsymbol{\theta}^{*}\right) \tau_{i j} \tau_{k l} .
$$

All functions are evaluated at the posterior mode $\theta^{*}$. The summations in 18 are over all subscripts and are from 1 to $d$, the dimension of the parameter vector $\theta$. The subscripts denote the partial derivative of the function with respect to the corresponding component of $\theta$, i.e., $g_{i j}=\partial^{2} g / \partial \theta_{i} \partial \theta_{j}$ and $\Lambda_{i j k}=\partial^{3} \Lambda / \partial \theta_{i} \partial \theta_{j} \partial \theta_{k}$, and $\left\{\tau_{i j}\right\}$ are the elements of the negative of the inverse of the Hessian matrix of $\Lambda$.

In our case, assuming $\theta=\left(\theta_{1}, \theta_{2}, \theta_{3}\right)=(\lambda, c, \theta)$, and with $g(\theta)=\lambda$, for example, equation (31) can be written as

$$
\begin{aligned}
\widehat{\lambda}_{L}= & \lambda^{*}+\frac{1}{2} \Lambda_{111} \tau_{11}^{2}+\frac{3}{2} \Lambda_{112} \tau_{11} \tau_{12}+\frac{3}{2} \Lambda_{113} \tau_{11} \tau_{13} \\
& +\frac{1}{2} \Lambda_{122}\left(2 \tau_{12}^{2}+\tau_{11} \tau_{22}\right)+\frac{1}{2} \Lambda_{123}\left(4 \tau_{12} \tau_{13}+2 \tau_{11} \tau_{23}\right) \\
& +\frac{1}{2} \Lambda_{133}\left(2 \tau_{13}^{2}+\tau_{11} \tau_{33}\right)+\frac{1}{2} \Lambda_{222} \tau_{12} \tau_{22} \\
& +\frac{1}{2} \Lambda_{223}\left(2 \tau_{12} \tau_{23}+\tau_{22} \tau_{13}\right)+\frac{1}{2} \Lambda_{233}\left(2 \tau_{13} \tau_{23}+\tau_{12} \tau_{33}\right) \\
& +\frac{1}{2} \Lambda_{333} \tau_{13} \tau_{33} .
\end{aligned}
$$

\section{Simulation Study}

In order to assess the performance of the proposed estimation methods (ML and Bayesian) under SRS and RSS schemes, a Monte Carlo simulation study of 5000 samples is conducted. Comparisons are made based on the bias, mean square error (MSE), and coverage probability (CP) and half length (HL) of 95\% confidence intervals. Different combinations of the parameter values are considered so as to cover different shapes of the probability density function of the GG distribution (see Figure 1). Since the conclusions were similar for almost all combinations of the parameter values, the results of the two combinations are presented, namely, $(\lambda, c, \theta)=(1,0.5,0.5)$ and $(\lambda, c, \theta)=(0.5,0.5,1)$. For each set of parameter values, 4 different sample sizes are studied, a small $n=10$, one moderate $n=20$, and two large $n=50$ and 100 sample sizes.

Bayesian estimates are obtained using the DE-M-H method with $N=10$ chains, each having a length of 10000 . The first half of each chain is considered as burn-in. The results of the simulation study are summarized in Tables 1-8. The following are observed:

(i) Bayesian estimation using $\mathrm{RW}-\mathrm{M}-\mathrm{H}$ algorithm produces a coverage probability of a $95 \%$ credible interval lower than the nominal rate even for large sample sizes. This can be seen in Table 8. This is because that the RW-M-H method did not explore the domain of the posterior distributions of the parameters. The trace plot of the MCMC chain of length $10^{4}$ for a randomly selected sample of size $n=$ 10 is shown in Figure 2. It can be seen from this plot that the DE-M-H chain covers a wider range of parameter values than the one obtained using RW$\mathrm{M}-\mathrm{H}$. Therefore, DE-M-H is used in our simulations to obtain Bayesian estimates and credible intervals.

(ii) Bayesian estimation using DE-M-H has better coverage probability, and it still has a lower MSE than the RW-M-H.

(iii) For a small sample size, the Bayesian approach produces a smaller MSE than the ML method. This is true under both RSS and SRS.

(iv) For moderate sample sizes, the ML methods produce a smaller MSE than the Bayesian method.

(v) In terms of the coverage probability (CP) and half length (HL) of the confidence interval, Bayesian approach produces better results (having more accurate $\mathrm{CP}$ and smaller HL) than the normal approximation of the ML approach under small and moderate sample sizes.

(vi) Confidence interval constructed using bootstrapping methods provides more accurate coverage probability with shorter half length compared with confidence intervals obtained using normal approximation for small and moderate sample sizes. Both methods perform almost the same as the sample size increases.

(vii) Bootstrapping confidence intervals were very comparable to Bayesian credible intervals in terms of half length and coverage probability.

(viii) DE-MCMC method provided smaller MSE than Lindley's method for small samples. Both methods performed almost the same for larger sample sizes.

(ix) The performance of both Bayesian and ML methods improves when using RSS compared with SRS even for small $m$. The performance improves as $m$ increases. 


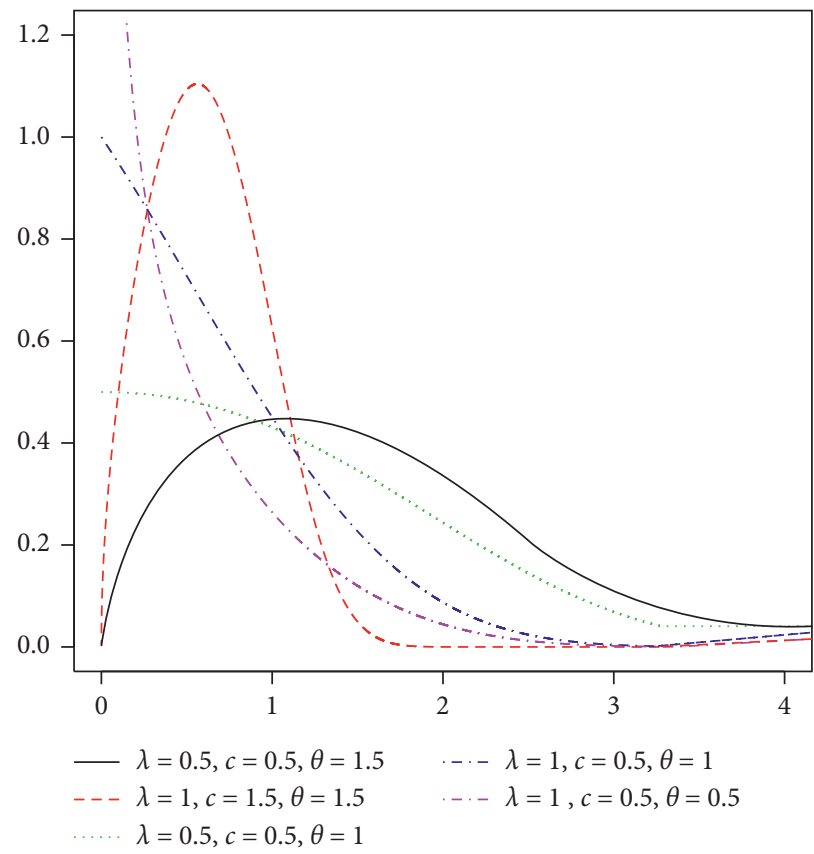

FIGURE 1: Shapes of the probability density function of GG distribution for different parameter values.

TABLE 1: Bias and MSE of the MLE and Bayes estimates under RSS and SRS for different sample sizes and different set sizes when $\lambda=0.5$, $c=0.5$, and $\theta=1.0$.

\begin{tabular}{|c|c|c|c|c|c|c|c|c|c|c|c|c|c|c|}
\hline \multirow[b]{3}{*}{$n$} & \multirow[b]{3}{*}{ Sampling } & \multirow[b]{3}{*}{$m$} & \multicolumn{4}{|c|}{$\lambda$} & \multicolumn{4}{|c|}{$c$} & \multicolumn{4}{|c|}{$\theta$} \\
\hline & & & \multicolumn{2}{|c|}{ MLE } & \multicolumn{2}{|c|}{ Bayes } & \multicolumn{2}{|c|}{ MLE } & \multicolumn{2}{|c|}{ Bayes } & \multicolumn{2}{|c|}{ MLE } & \multicolumn{2}{|c|}{ Bayes } \\
\hline & & & Bias & MSE & Bias & MSE & Bias & MSE & Bias & MSE & Bias & MSE & Bias & MSE \\
\hline \multirow{3}{*}{10} & SRS & & 0.140 & 0.392 & 0.405 & 0.392 & 0.403 & 1.015 & 0.101 & 0.490 & 0.587 & 2.745 & 1.025 & 2.556 \\
\hline & \multirow{2}{*}{ RSS } & 2 & 0.118 & 0.335 & 0.371 & 0.313 & 0.337 & 0.778 & 0.046 & 0.363 & 0.467 & 1.286 & 0.836 & 1.644 \\
\hline & & 5 & 0.066 & 0.224 & 0.312 & 0.229 & 0.298 & 0.638 & 0.028 & 0.345 & 0.227 & 0.943 & 0.520 & 1.177 \\
\hline \multirow{3}{*}{20} & SRS & & 0.068 & 0.173 & 0.325 & 0.237 & 0.175 & 0.298 & -0.055 & 0.249 & 0.186 & 0.608 & 0.446 & 0.877 \\
\hline & \multirow{2}{*}{ RSS } & 2 & 0.055 & 0.149 & 0.305 & 0.209 & 0.156 & 0.258 & -0.074 & 0.220 & 0.151 & 0.419 & 0.410 & 0.644 \\
\hline & & 5 & 0.030 & 0.109 & 0.263 & 0.166 & 0.134 & 0.202 & -0.081 & 0.187 & 0.092 & 0.241 & 0.332 & 0.391 \\
\hline \multirow{3}{*}{50} & SRS & & 0.023 & 0.060 & 0.221 & 0.128 & 0.064 & 0.079 & -0.108 & 0.104 & 0.059 & 0.114 & 0.233 & 0.213 \\
\hline & \multirow{2}{*}{ RSS } & 2 & 0.017 & 0.051 & 0.201 & 0.112 & 0.059 & 0.072 & -0.104 & 0.098 & 0.045 & 0.090 & 0.217 & 0.180 \\
\hline & & 5 & 0.011 & 0.040 & 0.168 & 0.088 & 0.050 & 0.059 & -0.092 & 0.082 & 0.030 & 0.067 & 0.182 & 0.133 \\
\hline \multirow{3}{*}{100} & SRS & & 0.007 & 0.027 & 0.118 & 0.063 & 0.034 & 0.035 & -0.059 & 0.054 & 0.024 & 0.043 & 0.118 & 0.082 \\
\hline & \multirow{2}{*}{ RSS } & 2 & 0.010 & 0.024 & 0.108 & 0.054 & 0.028 & 0.031 & -0.057 & 0.048 & 0.022 & 0.039 & 0.111 & 0.074 \\
\hline & & 5 & 0.004 & 0.019 & 0.080 & 0.039 & 0.026 & 0.027 & -0.041 & 0.039 & 0.015 & 0.029 & 0.087 & 0.053 \\
\hline
\end{tabular}

TABLE 2: Bias and MSE of the MLE and Bayes estimates under RSS and SRS for different sample sizes and different set sizes when $\lambda=1.0$, $c=0.5$, and $\theta=0.5$.

\begin{tabular}{|c|c|c|c|c|c|c|c|c|c|c|c|c|c|c|}
\hline \multirow[b]{3}{*}{$n$} & \multirow[b]{3}{*}{ Sampling } & \multirow[b]{3}{*}{$m$} & \multicolumn{4}{|c|}{$\lambda$} & \multicolumn{4}{|c|}{$c$} & \multicolumn{4}{|c|}{$\theta$} \\
\hline & & & \multicolumn{2}{|c|}{ MLE } & \multicolumn{2}{|c|}{ Bayes } & \multicolumn{2}{|c|}{ MLE } & \multicolumn{2}{|c|}{ Bayes } & \multicolumn{2}{|c|}{ MLE } & \multicolumn{2}{|c|}{ Bayes } \\
\hline & & & Bias & MSE & Bias & MSE & Bias & MSE & Bias & MSE & Bias & MSE & Bias & MSE \\
\hline \multirow{3}{*}{10} & SRS & & 0.080 & 1.139 & 0.487 & 1.148 & 1.146 & 5.389 & 0.402 & 1.795 & 0.084 & 0.185 & 0.145 & 0.211 \\
\hline & \multirow{2}{*}{ RSS } & 2 & 0.050 & 0.886 & 0.421 & 0.809 & 0.953 & 3.707 & 0.323 & 1.327 & 0.065 & 0.138 & 0.127 & 0.147 \\
\hline & & 5 & -0.039 & 0.535 & 0.300 & 0.459 & 0.832 & 2.918 & 0.260 & 1.240 & 0.022 & 0.051 & 0.081 & 0.057 \\
\hline \multirow{3}{*}{20} & SRS & & 0.022 & 0.449 & 0.363 & 0.452 & 0.481 & 1.090 & 0.005 & 0.454 & 0.032 & 0.040 & 0.079 & 0.046 \\
\hline & \multirow{2}{*}{ RSS } & 2 & 0.006 & 0.372 & 0.320 & 0.372 & 0.430 & 0.925 & -0.005 & 0.423 & 0.023 & 0.031 & 0.071 & 0.036 \\
\hline & & 5 & -0.029 & 0.271 & 0.253 & 0.257 & 0.370 & 0.700 & -0.035 & 0.328 & 0.008 & 0.019 & 0.055 & 0.022 \\
\hline \multirow{3}{*}{50} & SRS & & 0.008 & 0.172 & 0.280 & 0.217 & 0.170 & 0.241 & -0.152 & 0.160 & 0.012 & 0.012 & 0.049 & 0.015 \\
\hline & \multirow{2}{*}{ RSS } & 2 & -0.002 & 0.148 & 0.240 & 0.184 & 0.158 & 0.223 & -0.133 & 0.164 & 0.008 & 0.010 & 0.044 & 0.012 \\
\hline & & 5 & -0.008 & 0.112 & 0.207 & 0.140 & 0.134 & 0.180 & -0.139 & 0.141 & 0.003 & 0.007 & 0.038 & 0.008 \\
\hline \multirow{3}{*}{100} & SRS & & 0.002 & 0.087 & 0.223 & 0.144 & 0.085 & 0.101 & -0.156 & 0.112 & 0.006 & 0.005 & 0.036 & 0.007 \\
\hline & \multirow{2}{*}{ RSS } & 2 & 0.010 & 0.075 & 0.198 & 0.121 & 0.070 & 0.090 & -0.142 & 0.103 & 0.005 & 0.005 & 0.033 & 0.006 \\
\hline & & 5 & -0.004 & 0.054 & 0.159 & 0.089 & 0.068 & 0.077 & -0.126 & 0.093 & 0.002 & 0.003 & 0.029 & 0.005 \\
\hline
\end{tabular}


TABLE 3: Bias and MSE of the Bayes estimates obtained using DE-MCMC and Lindely methods under RSS and SRS for different sample sizes and different set sizes when $\lambda=1.0, c=0.5$, and $\theta=0.5$.

\begin{tabular}{|c|c|c|c|c|c|c|c|c|c|c|c|c|c|c|}
\hline \multirow[b]{3}{*}{$n$} & \multirow[b]{3}{*}{ Sampling } & \multirow[b]{3}{*}{$m$} & \multicolumn{4}{|c|}{$\lambda$} & \multicolumn{4}{|c|}{$c$} & \multicolumn{4}{|c|}{$\theta$} \\
\hline & & & \multicolumn{2}{|c|}{ MLE } & \multicolumn{2}{|c|}{ Bayes } & \multicolumn{2}{|c|}{ MLE } & \multicolumn{2}{|c|}{ Bayes } & \multicolumn{2}{|c|}{ MLE } & \multicolumn{2}{|c|}{ Bayes } \\
\hline & & & Bias & MSE & Bias & MSE & Bias & MSE & Bias & MSE & Bias & MSE & Bias & MSE \\
\hline \multirow{3}{*}{10} & SRS & & 0.487 & 1.148 & 0.463 & 1.498 & 0.402 & 1.795 & -0.536 & 1.509 & 0.145 & 0.211 & -0.638 & 0.812 \\
\hline & \multirow{2}{*}{ RSS } & 2 & 0.421 & 0.809 & -0.316 & 1.006 & 0.323 & 1.327 & 0.466 & 1.365 & 0.127 & 0.147 & 0.314 & 0.148 \\
\hline & & 5 & 0.3 & 0.459 & -0.283 & 0.584 & 0.26 & 1.24 & 0.431 & 1.198 & 0.081 & 0.057 & 0.543 & 0.468 \\
\hline \multirow{3}{*}{20} & SRS & & 0.363 & 0.452 & 0.201 & 0.473 & 0.005 & 0.454 & -0.158 & 0.453 & 0.079 & 0.046 & -0.518 & 0.331 \\
\hline & \multirow{2}{*}{ RSS } & 2 & 0.32 & 0.372 & -0.195 & 0.382 & -0.005 & 0.423 & 0.098 & 0.445 & 0.071 & 0.036 & 0.393 & 0.21 \\
\hline & & 5 & 0.253 & 0.257 & -0.188 & 0.242 & -0.035 & 0.328 & 0.077 & 0.327 & 0.055 & 0.022 & 0.312 & 0.211 \\
\hline \multirow{3}{*}{50} & SRS & & 0.28 & 0.217 & 0.161 & 0.218 & -0.152 & 0.16 & -0.067 & 0.171 & 0.049 & 0.015 & -0.262 & 0.169 \\
\hline & \multirow{2}{*}{ RSS } & 2 & 0.24 & 0.184 & -0.092 & 0.205 & -0.133 & 0.164 & 0.044 & 0.161 & 0.044 & 0.012 & 0.252 & 0.148 \\
\hline & & 5 & 0.207 & 0.14 & -0.082 & 0.178 & -0.139 & 0.141 & 0.025 & 0.15 & 0.038 & 0.008 & 0.245 & 0.139 \\
\hline \multirow{3}{*}{100} & SRS & & 0.223 & 0.144 & 0.073 & 0.168 & -0.156 & 0.112 & -0.025 & 0.117 & 0.036 & 0.007 & -0.18 & 0.118 \\
\hline & \multirow{2}{*}{ RSS } & 2 & 0.198 & 0.122 & -0.047 & 0.126 & -0.142 & 0.103 & 0.022 & 0.105 & 0.033 & 0.006 & 0.171 & 0.106 \\
\hline & & 5 & 0.159 & 0.089 & -0.045 & 0.086 & -0.126 & 0.093 & 0.018 & 0.095 & 0.029 & 0.005 & 0.078 & 0.084 \\
\hline
\end{tabular}

TABLE 4: Bias and MSE of the Bayes estimates obtained using DE-MCMC and Lindely methods under RSS and SRS for different sample sizes and different set sizes when $\lambda=0.5, c=0.5$, and $\theta=1$.

\begin{tabular}{|c|c|c|c|c|c|c|c|c|c|c|c|c|c|c|}
\hline \multirow[b]{3}{*}{$n$} & \multirow[b]{3}{*}{ Sampling } & \multirow[b]{3}{*}{$m$} & \multicolumn{4}{|c|}{$\lambda$} & \multicolumn{4}{|c|}{$c$} & \multicolumn{4}{|c|}{$\theta$} \\
\hline & & & \multicolumn{2}{|c|}{ MLE } & \multicolumn{2}{|c|}{ Bayes } & \multicolumn{2}{|c|}{ MLE } & \multicolumn{2}{|c|}{ Bayes } & \multicolumn{2}{|c|}{ MLE } & \multicolumn{2}{|c|}{ Bayes } \\
\hline & & & Bias & MSE & Bias & MSE & Bias & MSE & Bias & MSE & Bias & MSE & Bias & MSE \\
\hline \multirow{3}{*}{10} & SRS & & 0.405 & 0.392 & -0.138 & 0.368 & 0.101 & 0.49 & 0.365 & 0.456 & 1.025 & 2.556 & -0.169 & 2.093 \\
\hline & \multirow{2}{*}{ RSS } & 2 & 0.371 & 0.313 & -0.146 & 0.367 & 0.046 & 0.363 & 0.346 & 0.334 & 0.836 & 1.644 & -0.169 & 1.89 \\
\hline & & 5 & 0.312 & 0.229 & -0.13 & 0.306 & 0.028 & 0.345 & 0.307 & 0.328 & 0.52 & 1.177 & -0.138 & 0.85 \\
\hline \multirow{3}{*}{20} & SRS & & 0.325 & 0.237 & -0.088 & 0.266 & -0.055 & 0.249 & 0.227 & 0.253 & 0.446 & 0.877 & -0.11 & 0.89 \\
\hline & \multirow{2}{*}{ RSS } & 2 & 0.305 & 0.209 & -0.077 & 0.205 & -0.074 & 0.22 & 0.0196 & 0.217 & 0.41 & 0.644 & -0.084 & 0.683 \\
\hline & & 5 & 0.263 & 0.166 & -0.081 & 0.147 & -0.081 & 0.187 & 0.0181 & 0.184 & 0.332 & 0.391 & -0.08 & 0.371 \\
\hline \multirow{3}{*}{50} & SRS & & 0.221 & 0.128 & -0.038 & 0.138 & -0.108 & 0.104 & 0.1 & 0.106 & 0.233 & 0.213 & -0.042 & 0.251 \\
\hline & \multirow{2}{*}{ RSS } & 2 & 0.201 & 0.112 & -0.032 & 0.135 & -0.104 & 0.098 & 0.089 & 0.102 & 0.217 & 0.18 & -0.031 & 0.155 \\
\hline & & 5 & 0.168 & 0.088 & -0.031 & 0.086 & -0.092 & 0.082 & 0.077 & 0.081 & 0.182 & 0.133 & -0.032 & 0.139 \\
\hline \multirow{3}{*}{100} & SRS & & 0.118 & 0.063 & -0.019 & 0.067 & -0.059 & 0.054 & 0.052 & 0.053 & 0.118 & 0.082 & -0.019 & 0.095 \\
\hline & \multirow{2}{*}{ RSS } & 2 & 0.108 & 0.054 & -0.015 & 0.052 & -0.057 & 0.048 & 0.043 & 0.049 & 0.111 & 0.074 & -0.019 & 0.079 \\
\hline & & 5 & 0.08 & 0.039 & -0.017 & 0.035 & -0.041 & 0.039 & 0.042 & 0.039 & 0.086 & 0.053 & -0.015 & 0.053 \\
\hline
\end{tabular}

TABLE 5: Coverage probability (CP) and half length (HL) of the MLE and Bayes estimates under RSS and SRS for different sample sizes and different set sizes when $\lambda=0.5, c=0.5$, and $\theta=1.0$.

\begin{tabular}{|c|c|c|c|c|c|c|c|c|c|c|c|c|c|c|}
\hline \multirow[b]{3}{*}{$n$} & \multirow[b]{3}{*}{ Sampling } & \multirow[b]{3}{*}{$m$} & \multicolumn{4}{|c|}{$\lambda$} & \multicolumn{4}{|c|}{$c$} & \multicolumn{4}{|c|}{$\theta$} \\
\hline & & & \multicolumn{2}{|c|}{ MLE } & \multicolumn{2}{|c|}{ Bayes } & \multicolumn{2}{|c|}{ MLE } & \multicolumn{2}{|c|}{ Bayes } & \multicolumn{2}{|c|}{ MLE } & \multicolumn{2}{|c|}{ Bayes } \\
\hline & & & $\mathrm{CP}$ & $\mathrm{HL}$ & $\mathrm{CP}$ & $\mathrm{HL}$ & $\mathrm{CP}$ & $\mathrm{HL}$ & $\mathrm{CP}$ & $\mathrm{HL}$ & $\mathrm{CP}$ & $\mathrm{HL}$ & $\mathrm{CP}$ & $\mathrm{HL}$ \\
\hline \multirow{3}{*}{10} & SRS & & 0.822 & 1.409 & 0.951 & 1.013 & 0.970 & 1.752 & 0.949 & 1.201 & 0.908 & 3.555 & 0.949 & 3.253 \\
\hline & \multirow{2}{*}{ RSS } & 2 & 0.845 & 1.289 & 0.951 & 0.992 & 0.976 & 1.601 & 0.949 & 1.098 & 0.918 & 3.063 & 0.951 & 3.384 \\
\hline & & 5 & 0.840 & 1.037 & 0.950 & 0.775 & 0.978 & 1.377 & 0.949 & 1.037 & 0.910 & 1.695 & 0.950 & 1.647 \\
\hline \multirow{3}{*}{20} & SRS & & 0.880 & 0.831 & 0.949 & 0.706 & 0.994 & 0.994 & 0.951 & 0.902 & 0.935 & 1.216 & 0.951 & 1.165 \\
\hline & \multirow{2}{*}{ RSS } & 2 & 0.889 & 0.765 & 0.950 & 0.663 & 0.992 & 0.930 & 0.951 & 0.855 & 0.930 & 1.094 & 0.949 & 1.054 \\
\hline & & 5 & 0.891 & 0.652 & 0.951 & 0.589 & 0.986 & 0.820 & 0.951 & 0.793 & 0.931 & 0.887 & 0.949 & 0.877 \\
\hline \multirow{3}{*}{50} & SRS & & 0.922 & 0.473 & 0.950 & 0.521 & 0.968 & 0.533 & 0.949 & 0.596 & 0.950 & 0.596 & 0.948 & 0.657 \\
\hline & \multirow{2}{*}{ RSS } & 2 & 0.922 & 0.441 & 0.951 & 0.493 & 0.965 & 0.508 & 0.952 & 0.595 & 0.946 & 0.556 & 0.949 & 0.626 \\
\hline & & 5 & 0.927 & 0.387 & 0.950 & 0.445 & 0.961 & 0.460 & 0.952 & 0.556 & 0.949 & 0.483 & 0.950 & 0.551 \\
\hline \multirow{3}{*}{100} & SRS & & 0.928 & 0.323 & 0.947 & 0.395 & 0.959 & 0.357 & 0.951 & 0.463 & 0.951 & 0.392 & 0.949 & 0.466 \\
\hline & \multirow{2}{*}{ RSS } & 2 & 0.936 & 0.304 & 0.949 & 0.366 & 0.961 & 0.341 & 0.951 & 0.418 & 0.950 & 0.372 & 0.950 & 0.440 \\
\hline & & 5 & 0.934 & 0.268 & 0.950 & 0.332 & 0.956 & 0.313 & 0.948 & 0.410 & 0.953 & 0.328 & 0.950 & 0.384 \\
\hline
\end{tabular}


TABLE 6: Coverage probability (CP) and half length (HL) of the MLE and Bayes estimates under RSS and SRS for different sample sizes and different set sizes when $\lambda=1.0, c=0.5$, and $\theta=0.5$.

\begin{tabular}{|c|c|c|c|c|c|c|c|c|c|c|c|c|c|c|}
\hline \multirow[b]{3}{*}{$n$} & \multirow[b]{3}{*}{ Sampling } & \multirow[b]{3}{*}{$m$} & \multicolumn{4}{|c|}{$\lambda$} & \multicolumn{4}{|c|}{$c$} & \multicolumn{4}{|c|}{$\theta$} \\
\hline & & & \multicolumn{2}{|c|}{ MLE } & \multicolumn{2}{|c|}{ Bayes } & \multicolumn{2}{|c|}{ MLE } & \multicolumn{2}{|c|}{ Bayes } & \multicolumn{2}{|c|}{ MLE } & \multicolumn{2}{|c|}{ Bayes } \\
\hline & & & $\mathrm{CP}$ & $\mathrm{HL}$ & $\mathrm{CP}$ & $\mathrm{HL}$ & $\mathrm{CP}$ & $\mathrm{HL}$ & $\mathrm{CP}$ & $\mathrm{HL}$ & $\mathrm{CP}$ & $\mathrm{HL}$ & $\mathrm{CP}$ & $\mathrm{HL}$ \\
\hline \multirow{3}{*}{10} & SRS & & 0.818 & 2.297 & 0.976 & 2.410 & 0.979 & 3.368 & 0.963 & 1.759 & 0.931 & 0.680 & 0.961 & 0.939 \\
\hline & \multirow{2}{*}{ RSS } & 2 & 0.839 & 2.083 & 0.971 & 2.087 & 0.984 & 3.010 & 0.953 & 1.629 & 0.934 & 0.600 & 0.967 & 0.771 \\
\hline & & 5 & 0.833 & 1.642 & 0.969 & 1.643 & 0.983 & 2.524 & 0.949 & 1.463 & 0.928 & 0.443 & 0.959 & 0.557 \\
\hline \multirow{3}{*}{20} & SRS & & 0.874 & 1.438 & 0.950 & 1.311 & 0.986 & 1.743 & 0.950 & 1.079 & 0.950 & 0.371 & 0.950 & 0.409 \\
\hline & \multirow{2}{*}{ RSS } & 2 & 0.885 & 1.314 & 0.950 & 1.179 & 0.982 & 1.622 & 0.950 & 1.044 & 0.946 & 0.337 & 0.950 & 0.340 \\
\hline & & 5 & 0.886 & 1.095 & 0.950 & 0.949 & 0.976 & 1.420 & 0.950 & 0.951 & 0.945 & 0.277 & 0.950 & 0.268 \\
\hline \multirow{3}{*}{50} & SRS & & 0.920 & 0.856 & 0.949 & 0.792 & 0.970 & 0.902 & 0.952 & 0.705 & 0.957 & 0.210 & 0.949 & 0.209 \\
\hline & \multirow{2}{*}{ RSS } & 2 & 0.925 & 0.787 & 0.951 & 0.730 & 0.969 & 0.858 & 0.949 & 0.691 & 0.955 & 0.194 & 0.951 & 0.191 \\
\hline & & 5 & 0.934 & 0.674 & 0.951 & 0.635 & 0.969 & 0.778 & 0.951 & 0.656 & 0.955 & 0.164 & 0.950 & 0.162 \\
\hline \multirow{3}{*}{100} & SRS & & 0.929 & 0.593 & 0.949 & 0.618 & 0.961 & 0.596 & 0.952 & 0.573 & 0.959 & 0.144 & 0.949 & 0.148 \\
\hline & \multirow{2}{*}{ RSS } & 2 & 0.943 & 0.551 & 0.949 & 0.583 & 0.963 & 0.571 & 0.951 & 0.560 & 0.960 & 0.133 & 0.950 & 0.141 \\
\hline & & 5 & 0.947 & 0.471 & 0.950 & 0.501 & 0.958 & 0.523 & 0.950 & 0.538 & 0.955 & 0.114 & 0.951 & 0.120 \\
\hline
\end{tabular}

TABLE 7: Coverage probability (CP) and half length (HL) of the confidence intervals of the parameters using normal approximation and bootstrapping methods of the MLE estimates under RSS and SRS for different sample sizes and different set sizes when $\lambda=1.0, c=0.5$, and $\theta=0.5$.

\begin{tabular}{|c|c|c|c|c|c|c|c|c|c|c|c|c|c|c|}
\hline \multirow[b]{3}{*}{$n$} & \multirow[b]{3}{*}{ Sampling } & \multirow[b]{3}{*}{$m$} & \multicolumn{4}{|c|}{$\lambda$} & \multicolumn{4}{|c|}{$c$} & \multicolumn{4}{|c|}{$\theta$} \\
\hline & & & \multicolumn{2}{|c|}{ Normal } & \multicolumn{2}{|c|}{ Bottstrapping } & \multicolumn{2}{|c|}{ Normal } & \multicolumn{2}{|c|}{ Bootstrapping } & \multicolumn{2}{|c|}{ Normal } & \multicolumn{2}{|c|}{ Bootstrapping } \\
\hline & & & $\mathrm{CP}$ & $\mathrm{HL}$ & $\mathrm{CP}$ & HL & $\mathrm{CP}$ & $\mathrm{HL}$ & $\mathrm{CP}$ & $\mathrm{HL}$ & $\mathrm{CP}$ & $\mathrm{HL}$ & $\mathrm{CP}$ & $\mathrm{HL}$ \\
\hline \multirow{3}{*}{10} & SRS & & 0.818 & 2.297 & 0.993 & 2.113 & 0.979 & 3.368 & 0.948 & 4.197 & 0.931 & 0.68 & 0.992 & 1.122 \\
\hline & \multirow{2}{*}{ RSS } & 2 & 0.839 & 2.083 & 0.994 & 1.915 & 0.984 & 3.01 & 0.93 & 3.956 & 0.934 & 0.6 & 0.992 & 0.858 \\
\hline & & 5 & 0.833 & 1.642 & 0.989 & 1.448 & 0.983 & 2.524 & 0.908 & 3.094 & 0.928 & 0.443 & 0.98 & 0.545 \\
\hline \multirow{3}{*}{20} & SRS & & 0.874 & 1.438 & 0.969 & 1.278 & 0.986 & 1.743 & 0.917 & 1.938 & 0.95 & 0.371 & 0.973 & 0.417 \\
\hline & \multirow{2}{*}{ RSS } & 2 & 0.885 & 1.314 & 0.968 & 1.154 & 0.982 & 1.622 & 0.905 & 1.813 & 0.946 & 0.337 & 0.969 & 0.366 \\
\hline & & 5 & 0.886 & 1.095 & 0.958 & 0.961 & 0.976 & 1.42 & 0.906 & 1.541 & 0.945 & 0.277 & 0.975 & 0.284 \\
\hline \multirow{3}{*}{50} & SRS & & 0.92 & 0.856 & 0.971 & 0.775 & 0.97 & 0.902 & 0.93 & 0.914 & 0.957 & 0.21 & 0.96 & 0.21 \\
\hline & \multirow{2}{*}{ RSS } & 2 & 0.925 & 0.787 & 0.957 & 0.713 & 0.969 & 0.858 & 0.91 & 0.878 & 0.955 & 0.194 & 0.964 & 0.193 \\
\hline & & 5 & 0.934 & 0.674 & 0.96 & 0.626 & 0.969 & 0.778 & 0.935 & 0.788 & 0.955 & 0.164 & 0.958 & 0.161 \\
\hline \multirow{3}{*}{100} & SRS & & 0.929 & 0.593 & 0.953 & 0.558 & 0.961 & 0.596 & 0.934 & 0.593 & 0.959 & 0.144 & 0.964 & 0.141 \\
\hline & \multirow{2}{*}{ RSS } & 2 & 0.943 & 0.551 & 0.947 & 0.522 & 0.963 & 0.571 & 0.939 & 0.563 & 0.96 & 0.133 & 0.955 & 0.133 \\
\hline & & 5 & 0.947 & 0.471 & 0.956 & 0.45 & 0.958 & 0.523 & 0.932 & 0.517 & 0.955 & 0.114 & 0.949 & 0.112 \\
\hline
\end{tabular}

TABLE 8: Bias, MSE, coverage probability (CP), and half length (HL) of the Bayesian estimates based on DE-M-H and RW-M-H algorithms when $\lambda=0.5, c=0.5$, and $\theta=1.5$.

\begin{tabular}{|c|c|c|c|c|c|c|c|c|c|c|c|c|}
\hline \multirow[b]{3}{*}{$n$} & \multicolumn{4}{|c|}{$\lambda$} & \multicolumn{4}{|c|}{$c$} & \multicolumn{4}{|c|}{$\theta$} \\
\hline & \multicolumn{2}{|c|}{ DE-M-H } & \multicolumn{2}{|c|}{$\mathrm{M}-\mathrm{H}$} & \multicolumn{2}{|c|}{ DE-M-H } & \multicolumn{2}{|c|}{$\mathrm{M}-\mathrm{H}$} & \multicolumn{2}{|c|}{ DE-M-H } & \multicolumn{2}{|c|}{$\mathrm{M}-\mathrm{H}$} \\
\hline & Bias & MSE & Bias & MSE & Bias & MSE & Bias & MSE & Bias & MSE & Bias & MSE \\
\hline 20 & 0.372 & 0.287 & 0.333 & 0.364 & -0.061 & 0.235 & 0.018 & 0.526 & 1.091 & 4.639 & 0.97 & 5.745 \\
\hline 50 & 0.244 & 0.154 & 0.228 & 0.193 & -0.099 & 0.101 & -0.084 & 0.14 & 0.529 & 0.959 & 0.497 & 1.145 \\
\hline \multirow[t]{2}{*}{100} & 0.125 & 0.073 & 0.098 & 0.076 & -0.051 & 0.05 & -0.028 & 0.06 & 0.254 & 0.336 & 0.203 & 0.341 \\
\hline & $\mathrm{CP}$ & $\mathrm{HL}$ & $\mathrm{CP}$ & $\mathrm{HL}$ & $\mathrm{CP}$ & $\mathrm{HL}$ & $\mathrm{CP}$ & $\mathrm{HL}$ & $\mathrm{CP}$ & $\mathrm{HL}$ & $\mathrm{CP}$ & $\mathrm{HL}$ \\
\hline 20 & 0.95 & 0.737 & 0.812 & 0.538 & 0.949 & 0.879 & 0.822 & 0.721 & 0.95 & 2.422 & 0.858 & 1.895 \\
\hline 50 & 0.949 & 0.552 & 0.842 & 0.404 & 0.951 & 0.603 & 0.836 & 0.457 & 0.949 & 1.292 & 0.879 & 1.087 \\
\hline 100 & 0.942 & 0.407 & 0.861 & 0.299 & 0.949 & 0.438 & 0.865 & 0.332 & 0.948 & 0.881 & 0.898 & 0.726 \\
\hline
\end{tabular}



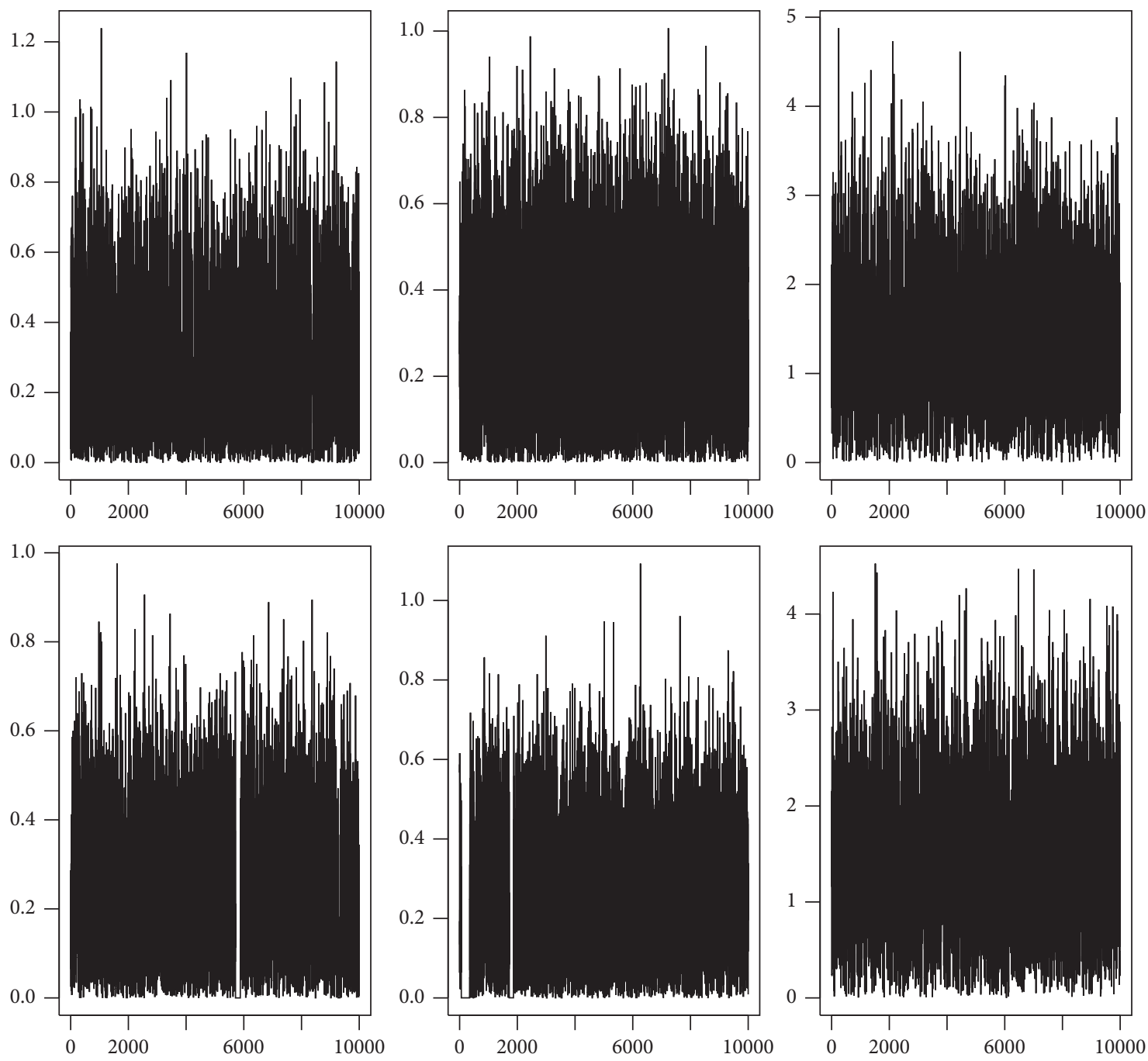

Figure 2: Trace plot of the posterior samples (length is $10^{4}$ ) of $\lambda$ (left), $c$ (middle), and $\theta$ (right) obtained using DE-M-H algorithm (top) and RW-M-H algorithm (bottom) for a randomly selected sample of size $n=100$ when $\lambda=0.5, c=0.5$, and $\theta=1.5$.

TABle 9: Failure time of 50 devices.

\begin{tabular}{|c|c|c|c|c|c|c|c|c|c|c|c|c|c|c|c|c|}
\hline 0.1 & 0.2 & 1 & 1 & 1 & 1 & 1 & 2 & 3 & 6 & 7 & 11 & 12 & 18 & 18 & 18 & 18 \\
\hline 18 & 21 & 32 & 36 & 40 & 45 & 45 & 47 & 50 & 55 & 60 & 63 & 63 & 67 & 67 & 67 & 67 \\
\hline 72 & 75 & 79 & 82 & 82 & 83 & 84 & 84 & 84 & 85 & 85 & 85 & 85 & 85 & 86 & 86 & \\
\hline
\end{tabular}

(x) As the sample size gets large, Bayesian and ML methods tend to perform almost the same under RSS and SRS.

In general, Bayesian approach is recommended for small sample sizes because it has better performance than the ML method. For moderate and large sample sizes, the ML approach is recommended due to its simplicity and speed. No matter which estimation method is used, we recommend using RSS over SRS as a sampling scheme because it has more efficient estimators even for small $m$.

\section{Real Data Example}

This section aims to implement and understand statistical inferential methods discussed in the previous sections via a real data example. The data considered are obtained from Aarset [21]. The data represent failure times of 50 devices obtained from a life-test experiment. These data were analyzed by many authors assuming GG distribution under different sampling schemes (see, for example, Ahmed [7] and El-Gohary et al. [4]). The GG distribution was found to fit the data fairly well and better than other candidate distributions such as Gompertz and generalized exponential and exponential distributions. The data are given in Table 9.

We assumed that the data in Table 9 to be our population. The true values of the parameters are obtained in ElGohary et al. [4] and are $\lambda=0.00143, c=0.044$, and $\theta=0.421$. To implement the proposed estimation methods, we analyzed 1000 bootstrap samples that were randomly 
TABLE 10: Mean and relative MSE of $\widehat{\theta}_{\mathrm{MLE}}$ and $\widehat{\theta}_{\mathrm{BAY}}$ for the real data example.

\begin{tabular}{|c|c|c|c|c|c|c|c|c|c|c|c|c|c|c|}
\hline \multirow[b]{3}{*}{$n$} & \multirow[b]{3}{*}{ Sampling } & \multirow[b]{3}{*}{$m$} & \multicolumn{4}{|c|}{$\lambda$} & \multicolumn{4}{|c|}{$c$} & \multicolumn{4}{|c|}{$\theta$} \\
\hline & & & \multicolumn{2}{|c|}{ MLE } & \multicolumn{2}{|c|}{ Bayes } & \multicolumn{2}{|c|}{ MLE } & \multicolumn{2}{|c|}{ Bayes } & \multicolumn{2}{|c|}{ MLE } & \multicolumn{2}{|c|}{ Bayes } \\
\hline & & & Mean & MSE & Mean & MSE & Mean & MSE & Mean & MSE & Mean & MSE & Mean & MSE \\
\hline \multirow{3}{*}{10} & SRS & & 0.004 & 0.017 & 0.004 & 0.019 & 0.034 & 0.004 & 0.055 & 0.002 & 0.632 & 0.331 & 0.609 & 0.319 \\
\hline & \multirow{2}{*}{ RSS } & 2 & 0.003 & 0.008 & 0.003 & 0.008 & 0.037 & 0.003 & 0.050 & 0.002 & 0.604 & 0.248 & 0.562 & 0.209 \\
\hline & & 5 & 0.003 & 0.006 & 0.003 & 0.005 & 0.039 & 0.002 & 0.049 & 0.002 & 0.604 & 0.231 & 0.495 & 0.243 \\
\hline \multirow{3}{*}{20} & SRS & & 0.002 & 0.004 & 0.003 & 0.004 & 0.038 & 0.001 & 0.050 & 0.002 & 0.558 & 0.181 & 0.456 & 0.070 \\
\hline & \multirow{2}{*}{ RSS } & 2 & 0.002 & 0.003 & 0.002 & 0.003 & 0.039 & 0.001 & 0.047 & 0.001 & 0.532 & 0.118 & 0.453 & 0.051 \\
\hline & & 5 & 0.002 & 0.002 & 0.002 & 0.002 & 0.041 & 0.001 & 0.047 & 0.001 & 0.504 & 0.135 & 0.444 & 0.0494 \\
\hline
\end{tabular}

TABLE 11: Coverage probability and half length of the confidence intervals for the real data example.

\begin{tabular}{|c|c|c|c|c|c|c|c|c|c|c|c|c|c|c|}
\hline \multirow[b]{3}{*}{$n$} & \multirow[b]{3}{*}{ Sampling } & \multirow[b]{3}{*}{$m$} & \multicolumn{4}{|c|}{$\lambda$} & \multicolumn{4}{|c|}{$c$} & \multicolumn{4}{|c|}{$\theta$} \\
\hline & & & \multicolumn{2}{|c|}{ MLE } & \multicolumn{2}{|c|}{ Bayes } & \multicolumn{2}{|c|}{ MLE } & \multicolumn{2}{|c|}{ Bayes } & \multicolumn{2}{|c|}{ MLE } & \multicolumn{2}{|c|}{ Bayes } \\
\hline & & & $\mathrm{CP}$ & HL & $\mathrm{CP}$ & $\mathrm{HL}$ & $\mathrm{CP}$ & $\mathrm{HL}$ & $\mathrm{CP}$ & HL & $\mathrm{CP}$ & $\mathrm{HL}$ & $\mathrm{CP}$ & $\mathrm{HL}$ \\
\hline \multirow{3}{*}{10} & SRS & & 0.963 & 0.006 & 0.933 & 0.006 & 0.957 & 0.023 & 0.935 & 0.019 & 0.96 & 0.522 & 0.95 & 0.561 \\
\hline & \multirow{2}{*}{ RSS } & 2 & 0.976 & 0.005 & 0.95 & 0.006 & 0.975 & 0.021 & 0.95 & 0.018 & 0.966 & 0.448 & 0.966 & 0.399 \\
\hline & & 5 & 0.986 & 0.005 & 0.959 & 0.005 & 0.986 & 0.019 & 0.965 & 0.016 & 0.975 & 0.394 & 0.968 & 0.345 \\
\hline \multirow{3}{*}{20} & SRS & & 0.969 & 0.003 & 0.947 & 0.004 & 0.969 & 0.017 & 0.949 & 0.016 & 0.933 & 0.330 & 0.95 & 0.337 \\
\hline & \multirow{2}{*}{ RSS } & 2 & 0.966 & 0.002 & 0.95 & 0.002 & 0.986 & 0.014 & 0.95 & 0.014 & 0.943 & 0.258 & 0.95 & 0.286 \\
\hline & & 5 & 0.971 & 0.002 & 0.949 & 0.002 & 0.939 & 0.013 & 0.948 & 0.013 & 0.947 & 0.237 & 0.949 & 0.283 \\
\hline
\end{tabular}

selected with replacement from the data. We considered two sample sizes $n=10,20$ and two values of the set size in RSS, $m=2,5$. The mean and relative MSE of the estimators and the half length and coverage probability of the confidence intervals are provided in Tables 10 and 11.

DE-MCMC method was used to obtain Bayesian estimates and credible interval. Bootstrapping confidence intervals were constructed for the ML estimates.

It is clear that the results of the real data agree with the simulation study. Under both SRS and RSS, the Bayes estimators outperform the MLE in terms of having lower MSE and lower half length of the confidence intervals. The two estimation methods perform almost similar as the sample size increases. The performance of the two estimation methods is improved when using RSS compared with SRS.

\section{Discussion and Conclusion}

This paper develops ML and Bayesian methods to estimate GG distribution parameters under RSS. The ML estimates are obtained, and the corresponding confidence interval of the parameters is obtained using both the normal approximation of the distribution of the ML estimates and bootstrapping methods. The Bayesian estimates are obtained under SEL function and weakly informative priors. It is observed that the Bayes estimators cannot be obtained in explicit forms, and therefore, differential evolution MCMC method is developed to obtain Bayesian point and interval estimates. Lindley's procedure is also studied to obtain Bayesian estimates. It was observed that Bayesian methods outperform the ML estimation methods in terms of MSE and coverage probability when the sample size is small. The opposite is true for moderate and large sample sizes. Under either method, estimates under RSS are more efficient than estimates under SRS.

\section{Data Availability}

The data used to support this study is obtained from the cited reference M. V. Aarset [21].

\section{Conflicts of Interest}

The authors declare that they have no conflicts of interest.

\section{Acknowledgments}

The first author acknowledges the Deanship of Scientific Research at King Faisal University for the financial support under Nasher Trach (Grant no. 186342).

\section{References}

[1] B. Gompertz, "On the nature of the function expressive of the law of human mortality, and on a new mode of determining the value of life contingencies," Philosophical Transactions of the Royal Society of London, vol. 115, pp. 513-583, 1825.

[2] H. M. Moustafa and S. G. Ramadan, "Errors of misclassification and their probability distributions when the parent populations are gompertz," Applied Mathematics and Computation, vol. 163, no. 1, pp. 423-442, 2005.

[3] M. M. M. El-Din, Y. Abdel-Aty, and M. H. Abu-Moussa, "Statistical inference for the gompertz distribution based on type-ii progressively hybrid censored data," Communications in Statistics - Simulation and Computation, vol. 46, no. 8, pp. 6242-6260, 2017.

[4] A. El-Gohary, A. Alshamrani, and A. N. Al-Otaibi, "The generalized gompertz distribution," Applied Mathematical Modelling, vol. 37, no. 1-2, pp. 13-24, 2013.

[5] P. Borges, "EM algorithm-based likelihood estimation for a generalized gompertz regression model in presence of survival data with long-term survivors: an application to uterine 
cervical cancer data," Journal of Statistical Computation and Simulation, vol. 87, no. 9, pp. 1712-1722, 2017.

[6] E. Demir and B. Saracoglu, "Maximum likelihood estimation for the parameters of the generalized gompertz distribution under progressive type-ii right censored samples," Journal of Selcuk University Natural and Applied Science, vol. 4, no. 1, pp. 41-48, 2015.

[7] E. A. Ahmed, "Estimation of some lifetime parameters of generalized gompertz distribution under progressively type-ii censored data," Applied Mathematical Modelling, vol. 39, no. 18, pp. 5567-5578, 2015.

[8] H. H. Abu-Zinadah and A. S. Al-Oufi, "Different method estimations of the three parameters of exponentiated gompertz distribution," Applied Mathematics \& Information Sciences, vol. 10, no. 2, pp. 705-710, 2016.

[9] H. H. Abu-Zinadah and A. S. Al-Oufi, "On estimations of parameters of generalized gompertz distribution," International Journal of Innovation in Science and Mathematics, vol. 3, no. 3, pp. 156-162, 2015.

[10] A. Martinez, "The defective generalized gompertz distribution and its use in the analysis of lifetime data in presence of cure fraction, censored data and covariates," Electronic Journal of Applied Statistical Analysis, vol. 10, no. 2, pp. 463-484, 2017.

[11] G. McIntyre, "A method for unbiased selective sampling, using ranked sets," Australian Journal of Agricultural Research, vol. 3, no. 4, pp. 385-390, 1952.

[12] V. Barnett and K. Moore, "Best linear unbiased estimates in ranked-set sampling with particular reference to imperfect ordering," Journal Of Applied Statistics, vol. 24, no. 6, pp. 697-710, 1997.

[13] D. A. Wolfe, "Ranked set sampling: its relevance and impact on statistical inference," ISRN Probability and Statistics, vol. 2012, Article ID 568385, 32 pages, 2012.

[14] J. Frey and Y. Zhang, "An algorithm with applications in ranked-set sampling," Journal of Statistical Computation and Simulation, vol. 88, no. 3, pp. 471-481, 2018.

[15] B. C. Arnold, N. Balakrishnan, and H. N. Nagaraja, A First Course in Order Statistics, John Wiley \& Sons, Hoboken, NJ, USA, 1992.

[16] J. F. Lawless, Statistical Models and Methods for Lifetime Data, John Wiley \& Sons, Hoboken, NJ, USA, 1982.

[17] B. Efron, "The jackknife, the bootstrap, and other resampling plans," Society for Industrial and Applied Mathematics, Philadelphia, PA, USA, 1982.

[18] A. Gelman, J. B. Carlin, H. S. Stern, and D. B. Rubin, Bayesian Data Analysis, Chapman \& Hall/CRC, Boca Raton, FL, USA, 2003.

[19] C. J. F. T. Braak, "A Markov chain Monte Carlo version of the genetic algorithm Differential Evolution: easy Bayesian computing for real parameter spaces," Statistics and Computing, vol. 16, no. 3, pp. 239-249, 2006.

[20] D. V. Lindley, "Approximate bayesian methods," Trabajos de Estadistica $Y$ de Investigacion Operativa, vol. 31, no. 1, pp. 223-245, 1980.

[21] M. V. Aarset, "How to identify a bathtub hazard rate," IEEE Transactions on Reliability, vol. R-36, no. 1, pp. 106-108, 1987. 